\title{
Maternal and fetal plasma platelet-activating factor acetylhydrolase activity and distribution in pre-eclampsia
}

\author{
Ping Fan ${ }^{1,2}$, Xing-Hui Liư ${ }^{3}$, Guo-Lin He ${ }^{3}$, Shuo Zhang ${ }^{3}$, Jin-Xia Zhang ${ }^{1,2}$ and Huai Bai1,2,
}

\begin{abstract}
BACKGROUND: Distribution of platelet-activating factor acetylhydrolase (PAF-AH) in lipoproteins plays important roles in the onset of inflammation and atherosclerosis. We hypothesized that women with pre-eclampsia (PE), showing signs of inflammation and oxidative stress, and their fetuses have aberrations of PAF-AH activity and distribution.
\end{abstract}

METHODS: Maternal and fetal plasma PAF-AH activity, highdensity lipoprotein (HDL)-associated PAF-AH (H-PAF-AH) and low-density lipoprotein (LDL)-associated PAF-AH (L-PAF-AH) were examined in women with PE $(n=127)$ and in women with uncomplicated pregnancies $(n=88)$.

RESULTS: The neonates of women with severe PE $(n=42)$ had significantly higher plasma PAF-AH, L-PAF-AH activities, and ratio of L-PAF-AH to H-PAF-AH activities than the neonates of women with normal pregnancies $(n=83)$. The mothers with severe PE $(n=106)$ and their neonates presented a significantly higher atherogenic index (Al) and triglyceride (TG)/HDL cholesterol (C) ratio than the control mothers and their neonates. The ratio of L-PAF-AH to H-PAF-AH activities correlated positively with TG levels, TG/HDL(C) ratio, and Al and negatively with $\mathrm{HDL}(\mathrm{C})$ levels in the neonates of women with PE.

CONCLUSION: The neonates of women with severe PE presented with a chronic inflammation status, increased oxidative stress, and unfavorable lipid changes, which may potentially link to related complications responsible for oxidative stress and inflammation in later life.

$\mathrm{P}$ re-eclampsia (PE) is a major cause of morbidity and mortality during pregnancy and childbirth. It is characterized by hypertension and an increased systemic inflammation response, potentially initiated by widespread endothelial damage and dysfunction (1-3). Patients with PE also demonstrate an increased oxidative stress, enhanced platelet aggregation and coagulation, and a marked dyslipidemia (1,4-7).

Recent evidence suggests that human fetuses exposed to an unfavorable uterine environment might permanently change their physiology and metabolism, and the programmed changes might be the origins of a number of diseases in later life, including cardiovascular disease, diabetes, hypertension, and polycystic ovary syndrome (PCOS) $(8,9)$. Exposure to PE has been shown to have an untoward effect in neonates on biochemical risk factor markers for cardiovascular disease including increased oxidative stress, enhanced plasma homocysteine and fibrinogen concentrations, and altered serum lipid profile $(1,10,11)$. There have been reports that the offspring exposed to PE in utero had higher systolic blood pressure and weighed more during adolescence, and the incidence of $\mathrm{PE}$ in later pregnancies was increased $(12,13)$.

Platelet-activating factor (PAF) is a biologically potent ether phospholipid generated by many types of cells including neutrophils, monocytes/macrophages, platelets, and endothelial cells (14). PAF-like oxidized phospholipids are oxidatively modified phospholipids that could be produced by oxidative stress. Both PAF and PAF-like oxidized phospholipids are potent proinflammatory mediators, and production and accumulation of these phospholipids are associated with the onset of inflammatory diseases such as asthma, sepsis, cardiac infarction, cerebral ischemia, and hypertension (15). Levels of PAF and malondialdehyde in maternal and/or fetal plasma in $\mathrm{PE}$ were elevated $(6,7,16)$, suggesting that PAF and oxidized lipids may contribute to the pathogenesis of $\mathrm{PE}$.

PAF acetylhydrolase (PAF-AH) hydrolyzes and inactivates PAF and PAF-like oxidized phospholipids. Plasma PAF-AH, also known as lipoprotein-associated phospholipase A2, is mainly bound to low-density lipoprotein (LDL) with a small portion also being associated with high-density lipoprotein (HDL) (15). Several studies showed associations between altered PAF-AH levels and cardiovascular disease or risk $(17,18)$ and the distribution of PAF-AH in lipoproteins plays an important role in atherosclerosis $(15,19,20)$. The alteration of PAF-AH activity is indicative of chronic inflammatory status $(21,22)$. The elevation of LDL-associated PAF-AH (L-PAF-AH) activity creates the conditions required for the formation of atherogenic small dense LDL and increases lysophosphatidylcholine and oxidatively modified nonesterified fatty acids with proatherogenic bioactivities through the oxidized LDL (15). In contrast, HDL-associated PAF-AH (H-PAF-AH) activity, although present at low levels in plasma, has been consistently shown to play an antiatherogenic

\footnotetext{
'Laboratory of Genetic Disease and Perinatal Medicine, West China Second University Hospital, Sichuan University, Sichuan, People's Republic of China; ${ }^{2}$ Key Laboratory of Obstetric and Gynecologic and Pediatric Diseases and Birth Defects of Ministry of Education, West China Second University Hospital, Sichuan University, Sichuan, People's Republic of China; ${ }^{3}$ Department of Obstetrics and Gynecology, West China Second University Hospital, Sichuan University, Sichuan, People's Republic of China. Correspondence: Huai Bai (baihuai60@yahoo.com.cn)
} 
role by helping to protect LDL from oxidation (15). It is now recognized that $\mathrm{H}-\mathrm{PAF}-\mathrm{AH}$ is the primary enzyme in $\mathrm{HDL}$ that is responsible for the hydrolysis of PAF and oxidized phospholipids (23). Our recent studies have demonstrated that the ratio of L-PAF-AH to H-PAF-AH activities was increased in patients with PCOS, a syndrome characterized by chronic inflammation $(24,25)$. It was suggested that the ratio of L-PAF-AH to $\mathrm{H}-\mathrm{PAF}-\mathrm{AH}$ activities might also represent a potential marker of inflammation and atherogenicity $(15,21,24)$.

Increased PAF-AH activity has been found in the maternal plasma and placentas in patients with PE $(26,27)$. However, the distribution of PAF-AH activity in lipoproteins in women with $\mathrm{PE}$ has not been reported yet, and the activity and distribution of the enzyme in fetal plasma is completely unknown. In this study, we hypothesized that women with PE, showing signs of inflammation and oxidative stress, and their fetuses would have aberrations of PAF-AH activity and distribution, which might contribute to the pathophysiology of PE and increased risk of cardiovascular disease in the patients and their offspring in later life. Therefore, we determined plasma PAF-AH, H-PAF-AH, and L-PAF-AH activities in maternal and fetal blood and analyzed the relationship between PAF-AH activities and clinical and metabolic parameters in patients with $\mathrm{PE}$.

\section{RESULTS}

\section{Clinical Characteristics of the Subjects}

As shown in Table 1, the levels of BMI, systolic blood pressure, and diastolic blood pressure were significantly higher in women with mild or severe PE as compared with healthy control women. Gestational age at sampling was lower in women with severe PE as compared with the controls or women with mild PE.

The mean fetal gestational age at delivery, birth height and weight of neonate, and Apgar score at $1 \mathrm{~min}$ were lower in the neonates of women with severe PE as compared with the neonates of women with normal pregnancies or mild PE (Table 1). There were no significant differences in these parameters between the neonates of women with mild PE and the neonates of women with normal pregnancies $(P>0.05)$.

\section{Plasma Lipid Profiles}

There were higher serum triglyceride (TG) levels, TG/HDL cholesterol $(\mathrm{C})$ ratio, and atherogenic index (AI), and lower $\mathrm{HDL}(\mathrm{C})$ levels in women with severe $\mathrm{PE}$ as compared with the controls. Women with mild PE also had significantly higher plasma TG levels and TG/HDL(C) ratio as compared with the controls (Table 2).

The neonates from women with severe PE had higher plasma TG, total cholesterol (TC) and LDL(C) levels, TG/HDL(C) ratio, and $\mathrm{AI}$ as compared with the neonates of normal pregnancies, and higher plasma $\operatorname{LDL}(\mathrm{C})$ levels as compared with the neonates from women with mild PE (Table 2).

\section{Plasma PAF-AH Activity and Its Distribution in Lipoproteins}

As shown in Table 3, the neonates of women with severe PE had significantly higher plasma PAF-AH and L-PAF-AH activities
Table 1. Clinical characteristics of mothers and neonates

\begin{tabular}{|c|c|c|c|}
\hline & Controls & $\begin{array}{l}\text { Mild } \\
\text { pre-eclampsia }\end{array}$ & $\begin{array}{c}\text { Severe } \\
\text { pre-eclampsia }\end{array}$ \\
\hline \multicolumn{4}{|l|}{ Maternal data } \\
\hline$n$ & 88 & 21 & 106 \\
\hline Maternal age $(y)$ & $29.3 \pm 4.9$ & $30.8 \pm 4.7$ & $29.6 \pm 5.8$ \\
\hline $\operatorname{BMI}\left(\mathrm{kg} / \mathrm{m}^{2}\right)$ & $27.0 \pm 2.3$ & $28.8 \pm 2.3^{*}$ & $28.5 \pm 3.2^{*}$ \\
\hline $\mathrm{SBP}(\mathrm{mm} \mathrm{Hg})$ & $115.1 \pm 10.2$ & $142.7 \pm 10.9^{*}$ & $164.1 \pm 20.9^{*}, * *$ \\
\hline $\mathrm{DBP}(\mathrm{mm} \mathrm{Hg})$ & $73.6 \pm 8.2$ & $90.1 \pm 7.8^{*}$ & $105.1 \pm 14.2^{*}, * *$ \\
\hline \multicolumn{4}{|l|}{ Parity } \\
\hline Nulliparous (\%) & 80.0 & 79.2 & 72.8 \\
\hline Multiparous (\%) & 20.0 & 20.8 & 27.4 \\
\hline $\operatorname{HELLP}(n)$ & 0 & 0 & 3 \\
\hline IUGR (n) & 0 & 3 & 36 \\
\hline $\begin{array}{l}\text { Gestation at } \\
\text { sampling (wk) }\end{array}$ & $38.4 \pm 2.1$ & $37.9 \pm 2.9$ & $34.8 \pm 4.2^{*},{ }^{* *}$ \\
\hline \multicolumn{4}{|l|}{ Neonatal data } \\
\hline$n$ & 83 & 13 & 42 \\
\hline Gestational age (wk) & $38.9 \pm 0.67$ & $38.0 \pm 1.3$ & $35.7 \pm 3.1^{* * *}$ \\
\hline$<34 \mathrm{wk}(n)$ & 0 & 0 & 15 \\
\hline$<37 \mathrm{wk}(n)$ & 0 & 4 & 27 \\
\hline \multicolumn{4}{|l|}{$\begin{array}{l}\text { Medications of } \\
\text { mothers }\end{array}$} \\
\hline $\mathrm{MgSO}_{4}(n)$ & 0 & 0 & 27 \\
\hline Steroids $(n)$ & 0 & 0 & 15 \\
\hline Birth height $(\mathrm{cm})$ & $49.0 \pm 1.9$ & $49.0 \pm 3.0$ & $44.4 \pm 4.9^{*},{ }^{* *}$ \\
\hline Birth weight (g) & $3,253 \pm 403$ & $3,098 \pm 568$ & $2,489 \pm 675^{* * *}$ \\
\hline $\begin{array}{l}\text { Apgar score }<7 \\
\text { at } 1 \min (n(\%))\end{array}$ & 0 & 0 & $8(19 \%)$ \\
\hline \multicolumn{4}{|c|}{ Values are presented as mean \pm SD. } \\
\hline \multicolumn{4}{|c|}{$\begin{array}{l}\text { DBP, diastolic blood pressure; HELLP, hemolysis, elevated liver enzymes, and low } \\
\text { platelets (syndrome); IUGR, intrauterine growth restriction; SBP, systolic blood pressure. }\end{array}$} \\
\hline \multicolumn{4}{|c|}{$\begin{array}{l}{ }^{*} P<0.05 \text {, compared with the control group; }{ }^{*} P<0.05 \text {, compared with the mild } \\
\text { pre-eclampsia group. }\end{array}$} \\
\hline
\end{tabular}

and ratio of L-PAF-AH to H-PAF-AH activities as compared with the neonates of normal pregnancies. However, there were no significant differences in plasma PAF-AH, H-PAF-AH, or L-PAF-AH activities or the ratio of L-PAF-AH to H-PAF-AH activities among the mothers with mild PE, mothers with severe PE, and control mothers $(P>0.05)$ (Table 3$)$.

Relationship Between PAF-AH Activity and Clinical and Metabolic Parameters in Women With PE and Their Neonates

Bivariate analysis showed that plasma PAF-AH and L-PAF-AH activities in women with PE correlated positively with TC $(r=0.339$ and 0.345 , respectively; $P<0.01), \mathrm{LDL}(\mathrm{C})(r=0.327$ and 0.340 , respectively; $P<0.01)$, and $\operatorname{HDL}(\mathrm{C})(r=0.189$ and 0.190 , respectively; $P<0.05$ ) levels and $\mathrm{H}-\mathrm{PAF}-\mathrm{AH}$ activity correlated positively with TC levels $(r=0.202 ; P<0.05)$. There was no significant correlation between $\mathrm{H}-\mathrm{PAF}-\mathrm{AH}$ activity and $\mathrm{HDL}(\mathrm{C})$ levels in the plasma of women with PE $(r=0.133 ; P>0.05)$. 
Table 2. Lipid profiles in mothers and neonates with mild and severe pre-eclampsia

\begin{tabular}{|c|c|c|c|}
\hline & \multirow[b]{2}{*}{ Controls } & \multicolumn{2}{|c|}{ Pre-eclampsia } \\
\hline & & Mild & Severe \\
\hline \multicolumn{4}{|l|}{ Mothers } \\
\hline $\mathrm{TG}(\mathrm{mmol} / \mathrm{l})$ & $3.38 \pm 1.35$ & $4.58 \pm 2.71^{*}$ & $3.98 \pm 1.69^{*}$ \\
\hline $\mathrm{TC}(\mathrm{mmol} / \mathrm{l})$ & $5.66 \pm 1.06$ & $6.04 \pm 1.57$ & $5.93 \pm 1.55$ \\
\hline $\mathrm{HDL}(\mathrm{C})(\mathrm{mmol} / \mathrm{l})$ & $1.58 \pm 0.42$ & $1.44 \pm 0.40$ & $1.37 \pm 0.48^{*}$ \\
\hline $\mathrm{LDL}(\mathrm{C})(\mathrm{mmol} / \mathrm{l})$ & $2.95 \pm 0.89$ & $2.73 \pm 1.08$ & $3.01 \pm 1.06$ \\
\hline Al & $2.78 \pm 1.13$ & $3.42 \pm 1.57$ & $3.68 \pm 1.57^{*}$ \\
\hline TG/HDL(C) & $2.28 \pm 1.05$ & $3.37 \pm 1.69^{*}$ & $3.38 \pm 2.28^{*}$ \\
\hline \multicolumn{4}{|l|}{ Neonates } \\
\hline $\mathrm{TG}(\mathrm{mmol} / \mathrm{l})$ & $0.18 \pm 0.08$ & $0.36 \pm 0.33$ & $0.57 \pm 0.35^{*}$ \\
\hline $\mathrm{TC}(\mathrm{mmol} / \mathrm{l})$ & $1.57 \pm 0.34$ & $1.68 \pm 0.42$ & $2.03 \pm 0.82^{*}$ \\
\hline $\mathrm{HDL}(\mathrm{C})(\mathrm{mmol} / \mathrm{l})$ & $0.78 \pm 0.18$ & $0.68 \pm 0.21$ & $0.69 \pm 0.35$ \\
\hline $\mathrm{LDL}(\mathrm{C})(\mathrm{mmol} / \mathrm{l})$ & $0.57 \pm 0.20$ & $0.62 \pm 0.23$ & $0.98 \pm 0.57^{*}, * *$ \\
\hline Al & $1.03 \pm 0.31$ & $1.59 \pm 0.73$ & $2.35 \pm 1.19^{*}$ \\
\hline $\mathrm{TG} / \mathrm{HDL}(\mathrm{C})$ & $0.26 \pm 0.18$ & $0.72 \pm 1.13$ & $1.41 \pm 1.59^{*}$ \\
\hline
\end{tabular}

Values are presented as mean \pm SD.

$\mathrm{Al}$, atherogenic index; C, cholesterol; HDL, high-density lipoprotein; LDL, low-density lipoprotein; TC, total cholesterol; TG, triglyceride.

${ }^{*} P<0.05$, compared with the control group after correction for gestational age in neonates or gestational age at sampling in mothers; ${ }^{* *} P<0.05$, compared with the mild pre-eclampsia group after correction for gestational age.

In the venous cord plasma of pregnancies complicated by $\mathrm{PE}$, the PAF-AH, L-PAF-AH, and H-PAF-AH activities correlated positively with plasma TC $(r=0.667,0.653$, and 0.639 , respectively; $P<0.01)$, $\mathrm{LDL}(\mathrm{C})(r=0.650,0.649$, and 0.552 , respectively; $P<0.01)$, and $\mathrm{HDL}(\mathrm{C})(r=0.493,0.462$, and 0.597 , respectively; $P<0.05)$ levels. The H-PAF-AH activity also correlated positively with placenta weight $(r=0.320 ; P<$ $0.05)$ and negatively with the TG/HDL(C) ratio $(r=-0.420 ; P$ $<0.01$ ), and the TG/HDL(C) ratio and AI correlated negatively with neonate's birth height $(r=-0.527$ and -0.783 , respectively; $P<0.05)$ and weight $(r=-0.446$ and -0.571 , respectively; $P$ $<0.01$ ), and the TG/HDL(C) ratio also correlated negatively with placenta weight $(r=-0.397 ; P<0.01)$.

\section{DISCUSSION}

Our major findings include the elevated plasma PAF-AH and L-PAF-AH activities, the increased ratio of L-PAF-AH to H-PAF-AH activities, and unfavorable proatherogenic lipoprotein ratios in the neonates of women with severe PE. To our knowledge, this is the first study reporting on the relationship between fetal plasma PAF-AH activity and distribution and PE.

The results of this study showing that L-PAF-AH and H-PAF-AH activities were positively correlated with LDL(C) and $\mathrm{HDL}(\mathrm{C})$ levels, respectively, in the venous cord plasma in women with $\mathrm{PE}$, are in line with our previous reports in patients with PCOS $(24,25)$. The elevation in the ratio of L-PAF-AH to $\mathrm{H}-\mathrm{PAF}-\mathrm{AH}$ activities might be associated with the increase in $\mathrm{LDL}(\mathrm{C})$ and/or decrease in $\mathrm{HDL}(\mathrm{C})$ levels. In addition,
Table 3. PAF-AH activities in mothers and neonates with mild and severe pre-eclampsia

\begin{tabular}{|c|c|c|c|}
\hline & \multirow[b]{2}{*}{ Controls } & \multicolumn{2}{|c|}{ Pre-eclampsia } \\
\hline & & Mild & Severe \\
\hline \multicolumn{4}{|l|}{ Mothers } \\
\hline $\begin{array}{l}\text { Plasma PAF-AH } \\
\text { (nmol/ml/min) }\end{array}$ & $45.16 \pm 13.54$ & $49.36 \pm 16.96$ & $45.35 \pm 14.64$ \\
\hline $\begin{array}{l}\text { H-PAF-AH } \\
\text { (nmol/ml/min) }\end{array}$ & $2.38 \pm 0.95$ & $3.04 \pm 1.61$ & $2.82 \pm 1.44$ \\
\hline $\begin{array}{l}\text { L-PAF-AH } \\
(\mathrm{nmol} / \mathrm{ml} / \mathrm{min})\end{array}$ & $42.77 \pm 12.86$ & $46.32 \pm 15.58$ & $42.53 \pm 13.57$ \\
\hline $\begin{array}{l}\text { L-PAF-AH/ } \\
\text { H-PAF-AH }\end{array}$ & $19.25 \pm 5.50$ & $17.28 \pm 5.51$ & $17.29 \pm 7.55$ \\
\hline \multicolumn{4}{|l|}{ Neonates } \\
\hline $\begin{array}{l}\text { Plasma PAF-AH } \\
\text { (nmol/ml/min) }\end{array}$ & $13.47 \pm 4.07$ & $15.36 \pm 4.55$ & $17.75 \pm 6.91^{*}$ \\
\hline $\begin{array}{l}\text { H-PAF-AH } \\
\text { (nmol/ml/min) }\end{array}$ & $1.61 \pm 0.58$ & $1.71 \pm 0.69$ & $1.91 \pm 1.01$ \\
\hline $\begin{array}{l}\text { L-PAF-AH } \\
\text { (nmol/ml/min) }\end{array}$ & $11.83 \pm 3.65$ & $13.65 \pm 3.98$ & $15.77 \pm 6.14^{*}$ \\
\hline $\begin{array}{l}\text { L-PAF-AH/ } \\
\text { H-PAF-AH }\end{array}$ & $7.69 \pm 1.91$ & $9.30 \pm 4.71$ & $9.26 \pm 3.48^{*}$ \\
\hline \multicolumn{4}{|c|}{ Values are presented as mean \pm SD. } \\
\hline \multicolumn{4}{|c|}{$\begin{array}{l}\text { H-PAF-AH, HDL-associated PAF-AH activity; LDL, low-density lipoprotein; L-PAF-AH, } \\
\text { LDL-associated-PAF-AH activity; PAF-AH, platelet-activating factor acetylhydrolase } \\
\text { activity. }\end{array}$} \\
\hline
\end{tabular}

this study showed that the ratio of L-PAF-AH to H-PAF-AH activities was positively correlated with serum TG levels and the TG/HDL(C) ratio, suggesting that serum TG levels also affected the distribution of PAF-AH activity between LDL and HDL in the venous cord plasma in PE. An increased ratio of L-PAF-AH to H-PAF-AH activities has also been reported in several other types of patients, such as those with hypercholesterolemia, combined hyperlipidemia or primary hypertriglyceridemia $(15,28)$, and those with atrial fibrillation or PCOS $(21,24,25)$. The elevated ratio of L-PAF-AH to H-PAF-AH activities might be a reflection of enhanced proinflammatory and proatherogenic changes in these patients $(15,21,24,25)$. Therefore, the increased ratio of L-PAF-AH to H-PAF-AH activities found in this study may reflect the chronic inflammation status and increased oxidative stress in the neonates of women with severe PE.

It has been recognized that some adult chronic diseases including cardiovascular disease might be associated with early-life exposures. PAF-AH might be one of the risk factors. Indeed, several studies have demonstrated that altered PAF-AH levels or activities, indicative of chronic inflammation status and/or oxidative stress, are associated with cardiovascular disease or risk $(21,22)$. For instance, Packard et al. (17) found increased levels of PAF-AH to be positively associated with the risk of coronary heart disease in a population of middle-aged men with hypercholesterolemia. There are reports that the distribution of PAF-AH in lipoproteins plays an important role in atherosclerosis $(15,19,20)$. The elevation 
of LDL-associated PAF-AH (L-PAF-AH) activity creates the conditions required for the formation of atherogenic small dense LDL and increases lysophosphatidylcholine and oxidatively modified nonesterified fatty acids with proatherogenic bioactivities through the oxidized LDL (15). Our study provides a potential link between altered PAF-AH levels and cardiovascular risk factors of the fetus, i.e., the neonates of the patients (especially the neonates of women with severe PE) might present a chronic inflammation status, and/or increased oxidative stress, in addition to an unfavorable lipid profile, and thus increase susceptibility to inflammatory diseases such as future cardiovascular diseases in later life, although the longterm effects of the increased ratio of L-PAF-AH to H-PAF-AH activities on the neonates of women with severe PE remain to be determined.

Ohshige et al. (29) reported increased PAF-AH activities in fetuses exposed to fetal growth restriction (venous cord plasma) as compared with appropriate growth for gestational age neonates. Our study showing variation of PAF-AH activities and distribution in the neonates of mothers with PE did not address whether there was any difference in PAF-AH activities between the neonates of the patients and neonates with growth restriction without PE. Further study including neonates with growth restriction without $\mathrm{PE}$ as controls will allow us to make such comparisons.

It has been reported that plasma PAF-AH activity was higher in women with $\mathrm{PE}(n=7)$ than in gestational age-matched normal controls $(n=24)(27)$. However, in this study, we did not find significant differences in plasma PAF-AH or L-PAF-AH activities or the ratio of L-PAF-AH to H-PAF-AH activities among the women with mild $\mathrm{PE}$, women with severe $\mathrm{PE}$, and the controls. A study has shown that plasma PAF-AH activity decreased significantly in maternal plasma during the latter stages of pregnancy in several species (30). The inconsistency of the results may be due to factors such as sample size and the difference of gestational age at sampling.

In addition, our study showed that unfavorable proatherogenic lipoprotein ratios, the $\mathrm{TG} / \mathrm{HDL}(\mathrm{C})$ ratio, and $\mathrm{AI}$ were more pronounced in women with severe $\mathrm{PE}$ and their neonates than in the respective controls. The cord venous blood TG/ $\mathrm{HDL}(\mathrm{C})$ ratio and AI correlated negatively with the neonate's birth height and weight, respectively, and the TG/HDL(C) ratio also correlated negatively with placenta weight in women with PE. The AI value represents ratio of "bad" cholesterol to "good" cholesterol in plasma, and increased AI is associated with increased risk of atherosclerosis, suggesting the potentially increased risk of atherosclerosis for the women with $\mathrm{PE}$ and their offspring. The TG/HDL(C) ratio has been identified to be the best predictor of insulin resistance and LDL particle diameter (31). A significantly elevated TG/HDL(C) ratio has been found to be associated with insulin resistance and increased small, dense, LDL particles in patients with metabolic syndrome and in women with PCOS (32). Because small, dense, LDL particles increase the susceptibility to augmented oxidation, and increased plasma lipid peroxides, including malondialdehyde levels, have been proven in patients with PE and their neonates $(6,10)$, the elevated TG/HDL(C) ratio may contribute to increased oxidative stress in mothers with severe $\mathrm{PE}$ and their neonates. We speculated that the increased TG/ $\mathrm{HDL}(\mathrm{C})$ ratio and AI might be potentially linked to the pathogenesis of PE and the increased cardiovascular risks in later life in the patients and their offspring.

We should point out that this study has some limitations. First, this study does not include women with chronic hypertension but no PE. Therefore, it is possible for maternal hypertension by itself to cause the neonatal changes. Second, based on the gestational age at delivery, it appears that most of the cases in this study are late-onset PE, which may be less relevant than early-onset disease. A control group that received medications such as magnesium sulfate and steroids would rule out the possible confounding effect of these agents.

In summary, this study showed that the neonates from women with severe PE have higher plasma PAF-AH and L-PAF-AH activities, higher ratio of L-PAF-AH to H-PAF-AH activities, and higher TG/HDL(C) ratio and AI than the neonates from women with normal pregnancies, suggesting that the neonates of the patients might present a chronic inflammation status, and/or increased oxidative stress, in addition to an unfavorable lipid profile. Whether these changes potentially link to the related complications responsible for the oxidative stress and inflammation in later life remains to be determined. These neonates born to women with PE deserve a closer clinical follow-up later in life.

\section{METHODS \\ Subjects}

One hundred and twenty-seven women with PE during 2006-2010 and 88 with uncomplicated pregnancies were studied, from whom 55 and 83 fetal samples were obtained, respectively. All study participants gave their informed consent and the study was approved by the institutional review board of the West China Second University Hospital, Sichuan University.

All patients with PE were recruited from the Department of Obstetrics and Gynecology of West China Second University Hospital at Sichuan University in Chengdu. PE was defined as systolic blood pressure of $>140 \mathrm{~mm} \mathrm{Hg}$ and/or diastolic blood pressure of $>90 \mathrm{~mm}$ $\mathrm{Hg}$ on two occasions $>6 \mathrm{~h}$ apart after $20 \mathrm{wk}$ of gestation, plus proteinuria of $>2+$ (dipstick method) or $>0.3 \mathrm{~g} / 24 \mathrm{~h}$; severe PE was defined as any one or more of the following occurring after the 20th week of pregnancy: (i) severe hypertension (systolic blood pressure $>160 \mathrm{~mm}$ $\mathrm{Hg}$ or diastolic blood pressure $>110 \mathrm{~mm} \mathrm{Hg}$ ); (ii) proteinuria $>5 \mathrm{~g} / 24 \mathrm{~h}$ or $>3+$ (dipstick method); (iii) oliguria $<500 \mathrm{ml} / 24 \mathrm{~h}$; (iv) cerebral irritability or visual disturbances; (v) epigastric or right upper quadrant pain; or (vi) pulmonary edema (33). The control women with uncomplicated pregnancies were recruited from the above department of the hospital during the same period.

Exclusion criteria for cases and controls were chronic hypertension, diabetes mellitus before/during pregnancy, cardiac disease, autoimmune disease, renal disease, and multifetal gestation. Women taking drugs known to affect lipid metabolism were excluded. Women with fetal growth restriction and their neonate(s) in the control group were also excluded.

Subjects were sampled at the time of recruitment to the study in the third trimester before delivery. Blood samples were obtained after an $8-12 \mathrm{~h}$ fast. Fetal cord blood was obtained at time of lower uterine cesarean section from the umbilical vein. Blood samples for PAF-AH activities were collected in tubes containing EDTA and centrifuged at $1500 \mathrm{~g}$ for $15 \mathrm{~min}$ within $2 \mathrm{~h}$. Plasma aliquots were stored at $-80^{\circ} \mathrm{C}$. 


\section{Analysis of Plasma Lipid Profile}

The concentrations of plasma TC, HDL(C), LDL(C), and TG were measured by enzymatic assay (Boehringer, Mannheim, Germany) using a Hitachi 7600-010 automatic analyzer. AI was calculated using the following equation: $\mathrm{AI}=(\mathrm{TC}-\mathrm{HDL}(\mathrm{C})) / \mathrm{HDL}(\mathrm{C})(34)$.

\section{Measurement of Plasma PAF-AH, H-PAF-AH, and L-PAF-AH Activities}

HDL fraction was separated by precipitating apoB-containing lipoproteins using the phosphotungstate- $\mathrm{Mg}^{2+}$ method $(35,36)$. Plasma PAF-AH and H-PAF-AH activities were measured by the trichloroacetic acid precipitation procedure as described previously (25). One unit of enzyme activity was defined as that which produced $1 \mathrm{nmol}$ of acetate per minute per $\mathrm{ml}$ plasma at $37^{\circ} \mathrm{C}$. The intra- and interassay coefficients of variation for all measurements were $<3$ and $5 \%$, respectively. L-PAF-AH activity was obtained by subtracting H-PAF-AH activity from plasma PAF-AH activity.

\section{Statistical Analysis}

Data are presented as mean \pm SD. Differences in variables were evaluated by ANOVA (least significant difference and Dunnett's T3) among the groups. Variables with asymmetric distribution were evaluated by nonparametric tests (Kruskal-Wallis $\mathrm{H}$ test). $\chi^{2}$ analysis was used to test deviations of parity between patients and controls. ANCOVA was used to estimate the differences of lipid parameters and PAF-AH activities among groups after correction for gestational age in neonates or gestational age at sampling in mothers. Correlations were performed using Pearson's coefficient of correlation. A value of $P<0.05$ was considered to be statistically significant. All statistical analyses were performed using Statistical Program for Social Sciences (SPSS) 13.0 for Windows (Chicago, IL).

\section{ACKNOWLEDGMENTS}

This work was performed at West China Second University Hospital, Sichuan University. We thank the patients with PE and control women who donated blood samples for this study.

\section{STATEMENT OF FINANCIAL SUPPORT}

This work was supported by grants from Research Seed Fund for returning scholars from the Ministry of Education, People's Republic of China (grant 20071108-18-3), Program for Changjiang Scholars and Innovative Research Team in University (grant IRT0935), Sichuan Province Science and Research Foundation of China (grant 2008SZ0029), and Research Seed Fund from West China Second University Hospital of Sichuan University (to H.B.).

\section{REFERENCES}

1. Rodie VA, Caslake MJ, Stewart F, et al. Fetal cord plasma lipoprotein status in uncomplicated human pregnancies and in pregnancies complicated by pre-eclampsia and intrauterine growth restriction. Atherosclerosis 2004;176:181-7.

2. Barden A, Ritchie J, Walters B, et al. Study of plasma factors associated with neutrophil activation and lipid peroxidation in preeclampsia. Hypertension 2001;38:803-8.

3. Roberts JM. Endothelial dysfunction in preeclampsia. Semin Reprod Endocrinol 1998;16:5-15.

4. Catarino C, Rebelo I, Belo L, et al. Fetal lipoprotein changes in pre-eclampsia. Acta Obstet Gynecol Scand 2008;87:628-34.

5. Laskowska M, Laskowska K, Leszczynska-Gorzelak B, Oleszczuk J. Comparative analysis of the maternal and umbilical interleukin-8 levels in normal pregnancies and in pregnancies complicated by preeclampsia with intrauterine normal growth and intrauterine growth retardation. J Matern Fetal Neonatal Med 2007;20:527-32.

6. Karabulut AB, Kafkasli A, Burak F, Gozukara EM. Maternal and fetal plasma adenosine deaminase, xanthine oxidase and malondialdehyde levels in pre-eclampsia. Cell Biochem Funct 2005;23:279-83.

7. Bulgan Kilicdag E, Ay G, Celik A, Ustundag B, Ozercan I, Simsek M. Oxidant-antioxidant system changes relative to placental-umbilical pathology in patients with preeclampsia. Hypertens Pregnancy 2005;24:147-57.
8. Barker DJ. A new model for the origins of chronic disease. Med Health Care Philos 2001;4:31-5.

9. Barker DJ. In utero programming of chronic disease. Clin Sci 1998; 95:115-28.

10. Howlader MZ, Parveen S, Tamanna S, Khan TA, Begum F. Oxidative stress and antioxidant status in neonates born to pre-eclamptic mother. J Trop Pediatr 2009;55:363-7.

11. Ophir E, Dourleshter G, Hirsh Y, Fait V, German L, Bornstein J. Newborns of pre-eclamptic women: a biochemical difference present in utero. Acta Obstet Gynecol Scand 2006;85:1172-8.

12. Vatten LJ, Romundstad PR, Holmen TL, Hsieh CC, Trichopoulos D, Stuver SO. Intrauterine exposure to preeclampsia and adolescent blood pressure, body size, and age at menarche in female offspring. Obstet Gynecol 2003;101:529-33.

13. Cooper DW, Hill JA, Chesley LC, Bryans CI. Genetic control of susceptibility to eclampsia and miscarriage. Br J Obstet Gynaecol 1988;95: 644-53.

14. Prescott SM, Zimmerman GA, Stafforini DM, McIntyre TM. Platelet-activating factor and related lipid mediators. Annu Rev Biochem 2000;69:419-45.

15. Karasawa K. Clinical aspects of plasma platelet-activating factoracetylhydrolase. Biochim Biophys Acta 2006;1761:1359-72.

16. Rowland BL, Vermillion ST, Roudebush WE. Elevated circulating concentrations of platelet activating factor in preeclampsia. Am J Obstet Gynecol 2000;183:930-2.

17. Packard CJ, O’Reilly DS, Caslake MJ, et al. Lipoprotein-associated phospholipase A2 as an independent predictor of coronary heart disease. West of Scotland Coronary Prevention Study Group. N Engl J Med 2000; 343:1148-55.

18. Caslake MJ, Packard CJ, Suckling KE, Holmes SD, Chamberlain P, Macphee $\mathrm{CH}$. Lipoprotein-associated phospholipase A(2), platelet-activating factor acetylhydrolase: a potential new risk factor for coronary artery disease. Atherosclerosis 2000;150:413-9.

19. Iribarren C. Lipoprotein-associated phospholipase A2 and cardiovascular risk: state of the evidence and future directions. Arterioscler Thromb Vasc Biol 2006;26:5-6.

20. Chen $\mathrm{CH}$. Platelet-activating factor acetylhydrolase: is it good or bad for you? Curr Opin Lipidol 2004;15:337-41.

21. Okamura K, Miura S, Zhang B, et al. Ratio of LDL- to HDL-associated platelet-activating factor acetylhydrolase may be a marker of inflammation in patients with paroxysmal atrial fibrillation. Circ J 2007;71:214-9.

22. Caslake MJ, Packard CJ. Lipoprotein-associated phospholipase A2 (platelet-activating factor acetylhydrolase) and cardiovascular disease. Curr Opin Lipidol 2003;14:347-52.

23. Kriska T, Marathe GK, Schmidt JC, McIntyre TM, Girotti AW. Phospholipase action of platelet-activating factor acetylhydrolase, but not paraoxonase-1, on long fatty acyl chain phospholipid hydroperoxides. J Biol Chem 2007;282:100-8.

24. Fan P, Liu HW, Wan DH, Li Y, Song Q, Bai H. Altered distribution of plasma platelet-activating factor acetylhydrolase between high-density lipoprotein and low-density lipoprotein in patients with polycystic ovary syndrome. Fertil Steril 2009;92:2054-7.

25. Fan P, Liu HW, Wang XS, et al. Identification of the G994T polymorphism in exon 9 of plasma platelet-activating factor acetylhydrolase gene as a risk factor for polycystic ovary syndrome. Hum Reprod 2010;25:1288-94.

26. Gu Y, Burlison SA, Wang Y. PAF levels and PAF-AH activities in placentas from normal and preeclamptic pregnancies. Placenta 2006;27: 744-9.

27. Maki N, Magness RR, Miyaura S, Gant NF, Johnston JM. Platelet-activating factor-acetylhydrolase activity in normotensive and hypertensive pregnancies. Am J Obstet Gynecol 1993;168(1 Pt 1):50-4.

28. Tsimihodimos V, Karabina SA, Tambaki AP, et al. Altered distribution of platelet-activating factor- acetylhydrolase activity between LDL and HDL as a function of the severity of hypercholesterolemia. J Lipid Res 2002;43:256-63.

29. Ohshige A, Yoshimura T, Maeda T, Ito M, Okamura H. Increased plateletactivating factor-acetylhydrolase activity in the umbilical venous plasma of growth-restricted fetuses. Obstet Gynecol 1999;93:180-3. 
30. Maki N, Hoffman DR, Johnston JM. Platelet-activating factor acetylhydrolase activity in maternal, fetal, and newborn rabbit plasma during pregnancy and lactation. Proc Natl Acad Sci USA 1988;85:728-32.

31. McLaughlin T, Reaven G, Abbasi F, et al. Is there a simple way to identify insulin-resistant individuals at increased risk of cardiovascular disease? Am J Cardiol 2005;96:399-404.

32. Shroff R, Syrop CH, Davis W, Van Voorhis BJ, Dokras A. Risk of metabolic complications in the new PCOS phenotypes based on the Rotterdam criteria. Fertil Steril 2007;88:1389-95.

33. Schroeder BM; American College of Obstetricians and Gynecologists. ACOG practice bulletin on diagnosing and managing preeclampsia and eclampsia. American College of Obstetricians and Gynecologists. Am Fam Physician 2002;66:330-1.
34. Demirel F, Bideci A, Cinaz P, et al. Serum leptin, oxidized low density lipoprotein and plasma asymmetric dimethylarginine levels and their relationship with dyslipidaemia in adolescent girls with polycystic ovary syndrome. Clin Endocrinol (Oxf) 2007;67: 129-34.

35. Zhang B, Fan P, Shimoji E, et al. Inhibition of cholesteryl ester transfer protein activity by JTT-705 increases apolipoprotein E-containing highdensity lipoprotein and favorably affects the function and enzyme composition of high-density lipoprotein in rabbits. Arterioscler Thromb Vasc Biol 2004;24:1910-5.

36. Chiba H, Akizawa K, Fujisawa S, et al. A rapid and simple quantification of human apolipoprotein E-rich high-density lipoproteins in serum. Biochem Med Metab Biol 1992;47:31-7. 